\title{
Light-by-light scattering in intense lasers
}

\section{B. King*}

Max-Planck-Institut für Kernphysik, Saupfercheckweg 1, D-69117 Heidelberg, Germany Arnold Sommerfeld Center for Theoretical Physics,

Ludwig-Maximilians-Universität München, Theresienstraße 37, 80333 München, Germany

E-mail: ben.king@physik.uni-muenchen.de

We present results of calculations for measuring the process of real photon-photon scattering using modern intense lasers. When a $10 \mathrm{PW}$ beam is split into two colliding pulses, we show that the elastic process is in principle measurable at upcoming laser facilities. If the pulses can be compressed into very few cycles, we show that the inelastic process, in which the frequencies of the two pulses are mixed, should also be measurable.

Photon 2013,

20-24 May 2013

Paris, France

${ }^{*}$ Speaker. 


\section{Introduction}

Since the founding of quantum mechanics, it has been long known that particles can interact via short-lived, so-called "virtual" states. To lowest order, photons, being mediators of electromagnetism, can interact with each other via virtual electron-positron pairs. Maxwell's equations describe the classical evolution of electromagnetic fields that are assumed not to interact with one another, which is expressed in the "principle of superposition". With the quantum correction due to virtual pairs, Maxwell's equations become augmented by a non-linear interaction between fields. For field inhomogeneities much larger than the dimension of a pair (assumed smaller than the length of field required to make the pair real), this non-linear interaction can be described by a result derived long ago by Heisenberg and Euler [1] and independently Weisskopf [2], following the pioneering work of Sauter [3], early note by Halpern [4] and work by Euler and Kockel [5]. The Heisenberg-Euler Lagrangian was then rederived as an effective theory of quantum electrodynamics (QED) by Schwinger [6]. Due to recent advances in the generation of high-intensity laser pulses [7], there has been renewed interest in measuring the predicted, although as yet unobserved, processes of QED in intense external fields, with one such example being real photon-photon scattering (a review of these processes can be found in [8,9]). Indeed, many methods have been devised to measure photon-photon scattering. For example, through the phase shift of one intense laser beam crossing another [10], the frequency shift of a photon propagating in an intense laser [11], in polarisation effects such as vacuum birefringence and dichroism [12,13,14, 15], dispersive effects such as vacuum diffraction $[16,17,18]$ and also in vacuum high harmonic generation [19]. Moreover, vacuum polarisation effects could potentially play an important role in the simulation of QED cascades [20,21]. Current experimental limits for photon-photon scattering [22] and vacuum birefringence [23], are still several orders higher than QED predicts, and such experiments are useful for excluding particle candidates for dark matter such as axions and WISPs [24, 25].

We present results of calculations for the scattering of real photons off of one another in the collision of intense laser pulses [26]. Due to the large number of photons involved, all fields are modelled as classical, and we consider the scattering of one probe laser pulse on a second, more intense, laser pulse. When the scattered and incoming spectra are identical, the process is referred to as "elastic", otherwise it originates from the four-wave mixing of the fields. The elastic process has already been investigated by considering the passage of one monochromatic Gaussian laser beam through another [27] and in so-called single- and "double-slit" set-ups [16, 12], in which a probe Gaussian beam diffracts off of others. Vacuum four-wave mixing has been investigated by considering $\mathrm{TE}_{10}$ and $\mathrm{TE}_{01}$ modes in a superconducting cavity [28] and in the collision of three, perpendicular, plane-waves [29]. In order to ascertain the suitability of measuring such processes in a laser-based experiment, we have calculated photon scattering for one of the most straightforward and realistic scenarios, namely the collision of two laser beams. By including many more experimental parameters than hitherto considered, such as pulse collision angle, lateral separation (impact parameter), lag (longitudinal phase difference), wavelength, focal width and pulse duration, we can better determine the possibility of measurement. As an example source of intense laser pulses, we have taken parameters around the 10 PW upgrade to the Vulcan Laser [30]. Unless otherwise apparent, we will work in a system of units with $\hbar=c=1$. 


\section{Envisaged experimental set-up}

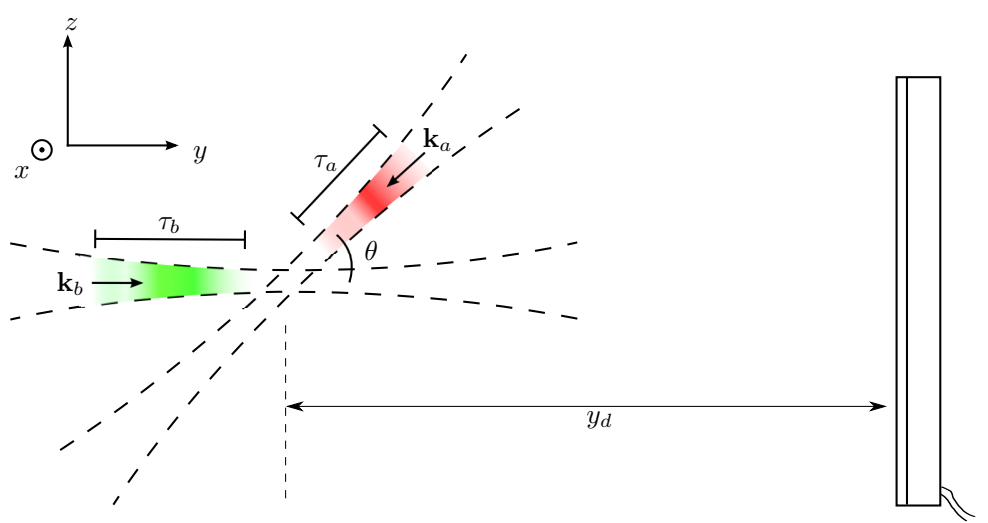

Figure 1: An example experimental set-up. $\tau_{a, b}$ are the pulse durations in the Gaussian beam envelopes $\mathrm{e}^{-(t \pm y)^{2} / \tau_{a, b}^{2}}$ and the stronger pulse $a$ is displaced from the origin in the probe pulse $b$ 's transverse plane by co-ordinates $x_{0}, z_{0}$. Both pulses have in general a carrier-envelope phase and the pulse $a$ lags behind $b$ by $\Delta t$. The scattered field and background from pulse $b$ are incident on the detector.

The calculated experimental scenario is shown in Fig. 1, in which two Gaussian laser pulses $a$ and $b$ of focal widths $w_{c, 0}$, durations $\tau_{c}$, frequencies $\omega_{c}$, wavelengths $\lambda_{c}$ and carrier envelope phases $\psi_{c}$, for $c \in\{a, b\}$, collide at an angle $\theta$, with lateral separation $x_{0}$ and $z_{0}$ along the $x$ and $z$ axes and lag $\Delta t$ to another, where the probe pulse $b$ propagates along the positive $y$ axis and whose centre at $t=0$ and carrier-envelope phase $\psi_{b}=0$ is at the origin. Specifically, the electric fields $\mathbf{E}_{c}$ are given by:

$$
\begin{aligned}
\mathbf{E}_{a}(x, y, z, t) & =\tilde{\boldsymbol{\varepsilon}}_{a} \frac{\mathscr{E}_{a} \mathrm{e}^{-\frac{\tilde{x}^{2}+z^{2}}{w_{a}^{2}(\tilde{y})}}}{\sqrt{1+\left(\tilde{y} / y_{r, a}\right)^{2}}} \sin \left[\psi_{a}+\omega_{a}(t-\Delta t+\tilde{y})-\eta_{a}(\tilde{y})\right] f_{a}(t-\Delta t+\tilde{y}) \\
\mathbf{E}_{b}(x, y, z, t) & =\hat{\boldsymbol{\varepsilon}}_{b} \frac{\mathscr{E}_{b} \mathrm{e}^{-\frac{x^{2}+z^{2}}{w_{b}^{2}(y)}}}{\sqrt{1+\left(y / y_{r, b}\right)^{2}}} \sin \left[\psi_{b}+\omega_{b}(t-y)+\eta_{b}(y)\right] f_{b}(t-y) \\
\eta_{c}(y) & =\tan ^{-1}\left(\frac{y}{y_{r, c}}\right)-\frac{\omega_{c} y}{2} \frac{x^{2}+z^{2}}{y^{2}+y_{r, c}^{2}}
\end{aligned}
$$

where $y_{r, c}=\omega_{c} w_{c, 0}^{2} / 2$ is the Rayleigh length, $w_{c}^{2}=w_{c, 0}^{2}\left[1+\left(y / y_{r, c}\right)^{2}\right]$ is the beam waist and the co-ordinates $(\tilde{x}, \tilde{y}, \tilde{z})$ are $(x, y, z)$ rotated anti-clockwise around the $x$ axis by an angle $\theta$, where the polarisation $\tilde{\boldsymbol{\varepsilon}}_{a}$ is similarly rotated so that $\mathbf{k}_{a} \cdot \hat{\boldsymbol{\varepsilon}}_{a}=\tilde{\mathbf{k}}_{b} \cdot \tilde{\boldsymbol{\varepsilon}}_{b}=0$ and $\left|\hat{\boldsymbol{\varepsilon}}_{a}\right|=\left|\tilde{\boldsymbol{\varepsilon}}_{b}\right|=1$, where $\mathbf{k}_{a}, \tilde{\mathbf{k}}_{b}$ are the beam wavevectors. We will specify:

$$
f_{c}(T)=\mathrm{e}^{-T^{2} / \tau_{c}^{2}},
$$

which corresponds to the lowest-order space and time approximation to the Gaussian solution, valid when $\omega_{c} \tau_{c} \gg 1$ and $w_{c, 0} / \lambda_{c, 0} \gg 1$. We will also assume that the magnetic fields obey $\mathbf{B}_{a}=\hat{\mathbf{k}}_{a} \wedge \mathbf{E}_{a}$, $\mathbf{B}_{b}=\hat{\tilde{\mathbf{k}}}_{b} \wedge \mathbf{E}_{b}, \hat{\tilde{\mathbf{k}}}_{a} \cdot \hat{\tilde{\mathbf{k}}}_{a}=\hat{\mathbf{k}}_{b} \cdot \hat{\mathbf{k}}_{b}=1$, which corresponds to dropping terms of the same order as neglected higher-order terms in the Gaussian solution. 


\section{Heisenberg-Euler Lagrangian}

The low-frequency $\left(\hbar \omega \ll m c^{2}\right.$ ) non-linear interaction due to the presence of virtual electronpositron pairs is calculated using the Heisenberg-Euler Lagrangian. This can be derived by considering the polarisation operator shown in Fig. 2 and integrating out the fermionic degrees of freedom to leave an "effective" interaction [6]. When the field strengths are such that $\left(E / E_{\text {cr }}\right)^{2} \ll 1$, where the critical field $E_{\mathrm{cr}}=m^{2} c^{3} / e \hbar$, one can perform a weak-field expansion of this Lagrangian. When the lowest order of this expansion is added to the classical Maxwellian Lagrangian, one has:

$$
\mathscr{L}=\frac{1}{8 \pi}\left(E^{2}-B^{2}\right)+\frac{1}{360 \pi^{2} E_{c r}^{2}}\left[\left(E^{2}-B^{2}\right)^{2}+7(\mathbf{E} \cdot \mathbf{B})^{2}\right] .
$$

As a $10 \mathrm{PW}$ laser is capable of producing intensities only of the order of $10^{24} \mathrm{Wcm}^{-2}$, corresponding to $\left(E / E_{\mathrm{cr}}\right)^{2} \sim 10^{-5}$, the weak-field expansion would appear justified.

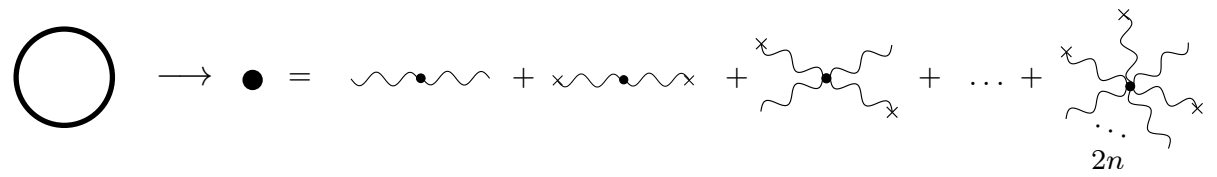

Figure 2: The Heisenberg-Euler Lagrangian is an integration over the high-energy (fermion) degrees of freedom of the polarisation operator (left) in a constant external field. The external field can be considered to originate from multiple sources, for example, photons from two sources are distinguished in the diagram, being with and without crosses.

From Eq. (3.1), we can derive a modified wave equation for electromagnetic fields:

$$
\begin{aligned}
\nabla^{2} \mathbf{E}-\partial_{t}^{2} \mathbf{E} & =4 \pi \mathbf{J}_{\mathrm{vac}}(E) \\
\mathbf{J}_{\mathrm{vac}} & =\left[\nabla \wedge \partial_{t} \mathbf{M}-\nabla(\nabla \cdot \mathbf{P})+\partial_{t}^{2} \mathbf{P}\right],
\end{aligned}
$$

where $\mathbf{P}=\frac{\partial \mathscr{L}}{\partial \mathbf{E}}-\frac{1}{4 \pi} \mathbf{E}, \mathbf{M}=\frac{\partial \mathscr{L}}{\partial \mathbf{B}}+\frac{1}{4 \pi} \mathbf{B}$ are the vacuum polarisation and magnetisation given by

$$
\begin{aligned}
\mathbf{P} & =\frac{\alpha^{2}}{180 \pi^{2} m^{4}}\left[2\left(E^{2}-B^{2}\right) \mathbf{E}+7(\mathbf{E} \cdot \mathbf{B}) \mathbf{B}\right] \\
\mathbf{M} & =-\frac{\alpha^{2}}{180 \pi^{2} m^{4}}\left[2\left(E^{2}-B^{2}\right) \mathbf{B}-7(\mathbf{E} \cdot \mathbf{B}) \mathbf{E}\right] .
\end{aligned}
$$

Since photon-photon scattering is a very weak process, in order to solve the implicit wave equation Eq. (3.2), we assume the vacuum current to be given, to a good approximation, by the polarising fields, thereby neglecting self-action terms:

$$
\begin{aligned}
\mathbf{E}(\mathbf{x}, t) & =\mathbf{E}_{0}(\mathbf{x}, t)+\mathbf{E}_{d}(\mathbf{x}, t) \\
\mathbf{E}_{d}(\mathbf{x}, t) & =\int d^{3} x^{\prime} \frac{\mathbf{J}_{\mathrm{vac}}\left[\mathbf{E}_{0}\left(\mathbf{x}^{\prime}, t-\left|\mathbf{x}-\mathbf{x}^{\prime}\right|\right)\right]}{\left|\mathbf{x}-\mathbf{x}^{\prime}\right|}
\end{aligned}
$$

where

$$
\nabla^{2} \mathbf{E}_{0}-\partial_{t}^{2} \mathbf{E}_{0}=0
$$


Although this quantum correction due to vacuum polarisation is calculated for classical fields, due to the large number of photons involved, we will interpret the scattered field in terms of numbers of photons. Indeed, the lowest-order weak-field expansion can be shown to be equivalent to the weak-field limit of the four-photon "box diagram" given in Fig. 3 [31] (a recent investigation of photon scattering in QED is given in [32]). The classical field then comprises a total number of photons $N_{t}$ equal to an integral of the field's spectrum divided by photon energy so that $N_{t}=\int_{-\infty}^{\infty} d \omega d x d z \widetilde{I}_{t}(\omega, \mathbf{x}) /|\omega|$, where the total spectral density $\widetilde{I}_{t}(\omega, \mathbf{x})=\left|\widetilde{\mathbf{E}}_{t}(\omega, \mathbf{x})\right|^{2} / 8 \pi^{2}=$ $\left|\widetilde{\mathbf{E}}_{b}(\omega, \mathbf{x})+\widetilde{\mathbf{E}}_{d}(\omega, \mathbf{x})\right|^{2} / 8 \pi^{2}\left(\widetilde{\mathbf{E}}_{a}(\omega, \mathbf{x})\right.$ is taken to be zero on the detector in the current set-up) and where $y$ is taken large enough that the surface perpendicular to the Poynting vector can be well approximated as being flat. The number of "measurable" photons corresponds to those incident on the detector in regions of high signal-to-noise ratio $N_{t}(x, z)-100 N_{b}(x, z)>0$ for $N_{i}(x, z)=$ $\int_{-\infty}^{\infty} d \omega \widetilde{I}_{i}(\omega, \mathbf{x}) /|\omega|, i \in\{a, b, d, t\}$.
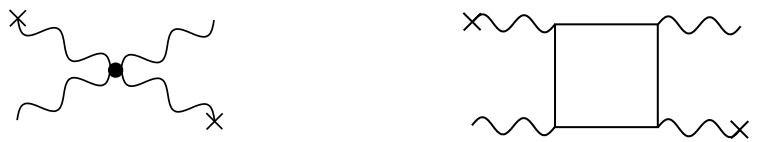

Figure 3: The lowest-order quantum correction to Maxwell's equations from the weak-field expansion of the Heisenberg-Euler Lagrangian (left) agrees with the QED calculation of the "box diagram" (right) in the limit $\hbar \omega / m c^{2} \ll 1$.

\section{Elastic photon-photon scattering}

By considering different combinations of incoming and outgoing photon legs in the fourphoton diagram, the "elastic" process corresponds to the probe photon interacting with an electronpositron pair, which absorbs and emits a strong-field photon to give an outgoing photon of equal outgoing energy, but different wavevector and polarisation:

$$
\gamma\left(\omega_{1}, \mathbf{k}_{1}, \boldsymbol{\varepsilon}_{1}\right)+\gamma\left(\omega_{2}, \mathbf{k}_{2}, \boldsymbol{\varepsilon}_{2}\right) \rightarrow \gamma\left(\omega_{1}, \mathbf{k}_{1}^{\prime}, \boldsymbol{\varepsilon}_{1}^{\prime}\right)+\gamma\left(\omega_{2}, \mathbf{k}_{2}^{\prime}, \boldsymbol{\varepsilon}_{2}^{\prime}\right),
$$

where $\gamma$ represents a photon, $\omega_{i}$ its frequency, $\mathbf{k}_{i}$ its wavevector and $\boldsymbol{\varepsilon}_{i}$ its polarisation. Due to high-intensity lasers typically producing pulses with several cycles, this is the most accessible photon-photon scattering event.

As we are interested in experimental measurement, we present calculations of the number of measurable diffracted photons $N_{d}$. A numerical test of our results was provided using the parameters $\lambda_{a}=0.8 \mu \mathrm{m}, \lambda_{b}=0.527 \mu \mathrm{m}, w_{a, 0}=0.8 \mu \mathrm{m}, w_{b, 0}=290 \mu \mathrm{m}, P_{a}=50 \mathrm{PW}, P_{b}=20 \mathrm{TW}$ to find agreement with the head-on single-slit results from [33]. The number of measurable diffracted photons was then calculated for parameters typical of the Vulcan laser $\lambda_{a}=\lambda_{b}=0.91 \mu \mathrm{m}, \tau_{a}=$ $\tau_{b}=30 \mathrm{fs}, P_{a}=5 \mathrm{PW}, P_{b}=5 \mathrm{PW}$, with $w_{a, 0}=0.91 \mu \mathrm{m}, w_{b, 0}=100 \mu \mathrm{m}, \boldsymbol{\varepsilon}_{a}=\boldsymbol{\varepsilon}_{b}=\hat{\mathbf{x}}, \hat{\mathbf{x}} \cdot \hat{\mathbf{x}}=1$. Several parameters were individually varied, the results plotted in Fig. 4 and their dependencies summarised in Tab. 1. 


\begin{tabular}{ccc} 
Parameter & $N_{d}$ scaling & Range observed \\
\hline$w_{b, 0}$ & $w_{b, 0}^{-2}$ & $w_{b, 0}[\mu \mathrm{m}]>80$ \\
$x_{0}$ & $\exp \left[-2\left(x_{0} / w_{b, 0}\right)^{2}\right]$ & $\left|x_{0}\right| \leq 2 w_{b, 0}$ \\
$\lambda=\lambda_{a}=\lambda_{b}$ & $\lambda^{-3}$ & $0.2 \leq \lambda[\mu \mathrm{m}] \lesssim 0.5$ \\
& $\lambda^{-7 / 2}$ & $0.5 \lesssim \lambda[\mu \mathrm{m}] \leq 1.0$ \\
$\tau=\tau_{a}=\tau_{b}$ & $\tau^{3}$ & $5 \leq \tau[\mathrm{fs}] \lesssim 200$ \\
$\theta$ & $\tau$ & $10^{3} \lesssim \tau[\mathrm{fs}] \leq 2 \times 10^{4}$ \\
$\Delta t$ & $\approx(1+\cos \theta)^{2}$ & $\theta \leq \pi / 2$ \\
& $\exp \left[-(\Delta t / 9 \tau)^{2}\right]$ & $0 \leq \Delta \tau / \tau \leq 20$
\end{tabular}

Table 1: Constant parameters were $\lambda_{a}=\lambda_{b}=0.91 \mu \mathrm{m}, w_{a, 0}=0.91 \mu \mathrm{m}, w_{b, 0}=100 \mu \mathrm{m}, \tau_{a}=\tau_{b}=30 \mathrm{fs}$, $P_{a}=5 \mathrm{PW}, P_{b}=5 \mathrm{PW}, \boldsymbol{\varepsilon}_{a}=\boldsymbol{\varepsilon}_{b}=\hat{\mathbf{x}}$.

For the purposes of detection in experiment, there are a few methods that could be used to increase the number of diffracted photons. First, if the laser pulses' polarisation are chosen to be perpendicular, all results in Fig. 4 will be increased by approximately a factor 3. Second, one could use higher-harmonics of the probe laser. If the same parameters as in Fig. 2 are used, for a collision angle of $\theta=0.1$, assuming a $40 \%$ reduction in energy due to generating the second harmonic, $N_{d} \approx 4$. If this process could be repeated to generate the fourth-harmonic, with a $16 \%$ overall reduction, $N_{d} \approx 13$. It has been previously argued, (for example in [16]), that such low numbers of scattered photons should in principle be detectable in an experiment. A discussion of sources of background noise can be found in [16, 33].

\section{Four-wave mixing}

Instead of two incoming and outgoing photons, other combinations are possible and we label these processes "four-wave mixing". The quantised analogue of one of these processes is given by:

$$
\gamma\left(\omega_{1}, \mathbf{k}_{1}, \boldsymbol{\varepsilon}_{1}\right)+\gamma\left(\omega_{2}, \mathbf{k}_{2}, \boldsymbol{\varepsilon}_{2}\right) \rightarrow \gamma\left(\omega_{3}, \mathbf{k}_{3}, \boldsymbol{\varepsilon}_{3}\right)+\gamma\left(\omega_{4}, \mathbf{k}_{4}, \boldsymbol{\varepsilon}_{4}\right),
$$

where again, $\gamma$ represents a photon, $\omega_{i}$ its frequency, $\mathbf{k}_{i}$ its wavevector and $\boldsymbol{\varepsilon}_{i}$ its polarisation and the photons can take their parameters from any of the those offered by the pulses. Energy-momentum conservation implies $k=k_{1}+k_{2}+k_{3}$, where $k$ is the momentum of the scattered photon. Then considering different combinations, we find:

$$
\begin{aligned}
\omega= & \operatorname{sgn}\left(\beta_{j}\right)\left[\omega_{a, 1}+\delta_{\left|\beta_{j}\right| 2} \omega_{a, 2}\right]+\operatorname{sgn}\left(\gamma_{j}\right)\left[\omega_{b, 1}+\delta_{\left|\gamma_{j}\right| 2} \omega_{b, 2}\right] \\
\omega \frac{y}{r}= & \operatorname{sgn}\left(\beta_{j}\right)\left[\omega_{a, 1} \cos \theta_{a, 1}+\delta_{\left|\beta_{j}\right| 2} \omega_{a, 2} \cos \theta_{a, 2}\right] \\
& +\operatorname{sgn}\left(\gamma_{j}\right)\left[\omega_{b, 1} \cos \theta_{b, 1}+\delta_{\left|\gamma_{j}\right| 2} \omega_{b, 2} \cos \theta_{b, 2}\right] \\
\omega \frac{\rho}{r}= & \operatorname{sgn}\left(\beta_{j}\right)\left[\omega_{a, 1} \sin \theta_{a, 1}+\delta_{\left|\beta_{j}\right| 2} \omega_{a, 2} \sin \theta_{a, 2}\right] \\
& +\operatorname{sgn}\left(\gamma_{j}\right)\left[\omega_{b, 1} \sin \theta_{b, 1}+\delta_{\left|\gamma_{j}\right| 2} \omega_{b, 2} \sin \theta_{b, 2}\right],
\end{aligned}
$$

where $\omega$ is the frequency generated, $\rho^{2}=x^{2}+z^{2}, j$ runs from 1 to $12, \beta_{j}, \gamma_{j}$ are coefficients from Tab. 2: 

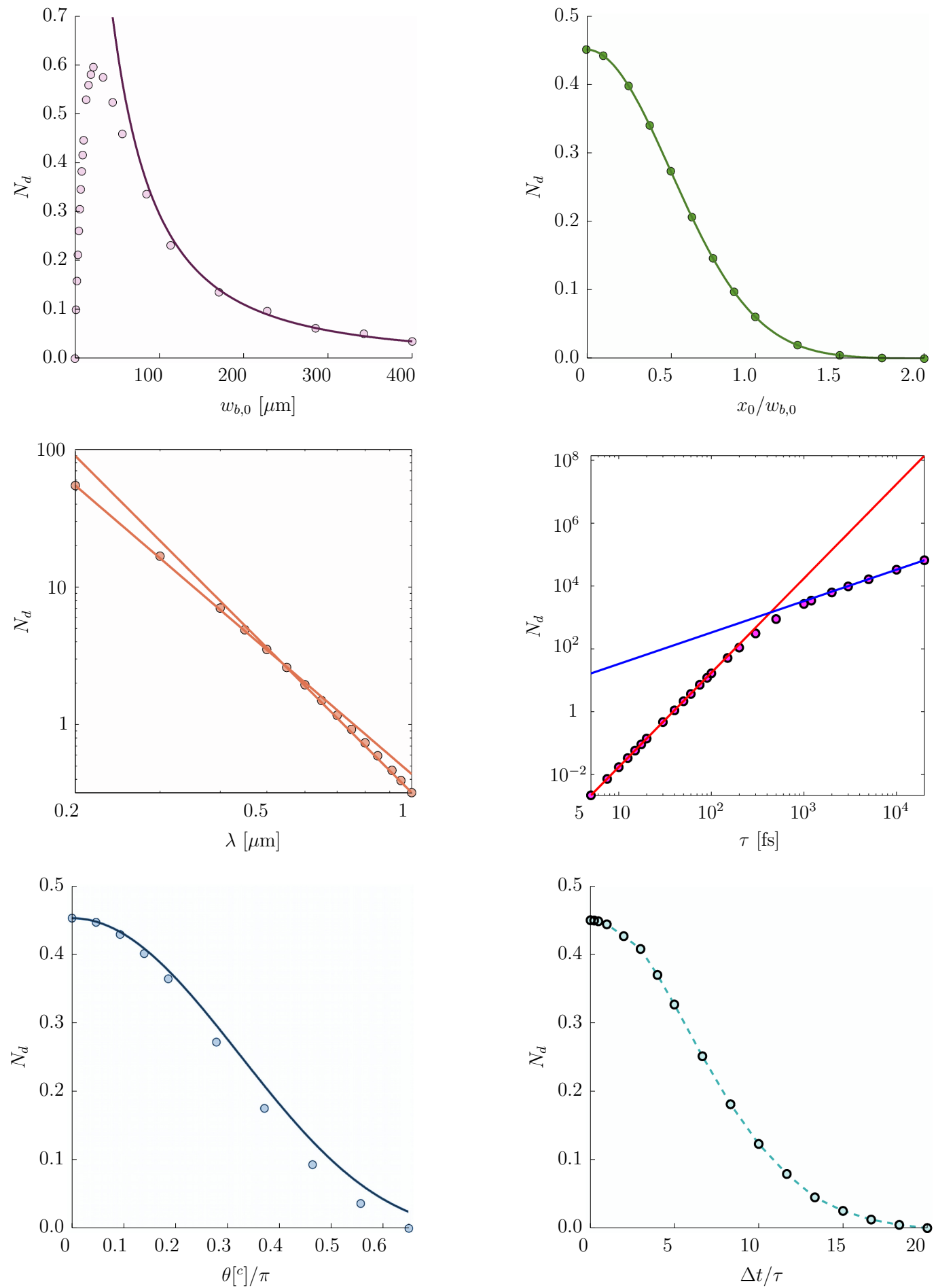

Figure 4: Number of measurable diffracted photons for the parameters $\lambda_{a}=\lambda_{b}=0.91 \mu \mathrm{m}, w_{a, 0}=0.91 \mu \mathrm{m}$, $w_{b, 0}=100 \mu \mathrm{m}, \tau_{a}=\tau_{b}=30 \mathrm{fs}, P_{a}=5 \mathrm{PW}, P_{b}=5 \mathrm{PW}, \hat{\boldsymbol{\varepsilon}}_{a}=\hat{\boldsymbol{\varepsilon}}_{b}=\hat{\mathbf{x}}$. The plot in $\tau$ expands upon the small data range plotted in [26], with the rest adapted from the same source. 


\begin{tabular}{r|rrrrrrrrrrrr}
$j$ & 1 & 2 & 3 & 4 & 5 & 6 & 7 & 8 & 9 & 10 & 11 & 12 \\
$\beta_{j}$ & 2 & -2 & 0 & 2 & -2 & 0 & 1 & 1 & 1 & -1 & -1 & -1 \\
$\gamma_{j}$ & 1 & 1 & 1 & -1 & -1 & -1 & 2 & -2 & 0 & 2 & -2 & 0
\end{tabular}

Table 2: Combinations of coefficients occurring in the energy-momentum relations for four-wave mixing,

$\theta_{c, i}$ are the angles the photons make with the positive $y$-direction and $\operatorname{sgn}(x)$ returns the sign of $x$ with $\operatorname{sgn}(0)=0$. Here we notice the correlation between harmonic order and detection co-ordinate, which can be compared to high-harmonic generation in plasmas [34]. It turns out to be difficult to satisfy these conditions simultaneously with just two laser beams and a fixed observation angle.

We investigated the ansatz that for short enough pulses, the bandwidth of the two lasers becomes wide enough that Eqs. (5.2-5.4) can be fulfilled simultaneously for a measurable amount of photons. Our calculations are restricted as to how short the pulse duration can be made, as the Gaussian temporal envelope solution is only a good approximation to Maxwell's equations when $\omega_{c} \tau_{c} \gg 1$. However, to produce intense laser pulses that are shorter than this is currently experimentally prohibitive. This lower bound limits our ability to assess the importance of the inelastic process since we require a large bandwidth $\Delta \omega / \omega$ for the photons generated via four-wave mixing to be on shell, but due to the bandwidth theorem, $\Delta \omega / \omega \sim 1 / \omega \tau \ll 1$ from our limitation on $\tau$. So instead of looking to separate off the four-wave mixing part of the scattered photons' spectrum, it seems more likely to be able to observe the change in the total number of diffracted photons due to this process. In Fig. 5, we plot the ratio $\left(N_{t}-N_{e}\right) / N_{e}$ against $\tau_{a}$, where $N_{e}$ is the number of elastically-scattered photons. As can be seen, for short enough pulse durations, the results suggest that the four-wave mixing process can visibly influence the total number of measured photons. For the quoted Vulcan laser parameters, in Fig. 5 the proportion reaches over $20 \%$, for a minimum pulse duration of $\tau=1 \mathrm{fs}$, equivalent to $\omega_{a} \tau_{a} \approx 2$. If one assumes a $40 \%$ attenuation each time a second-harmonic is generated from the probe laser pulse, the total number of measurable diffracted photons ranges from 1 to 4 (at $\tau_{a}=1,2$ fs respectively). Even though the analysis is restricted due to the shortness of the pulse, the effect was found to persist and increase also when two, $(1+1) D$ sech pulses were considered instead [26], and the pulse duration could be made arbitrarily small.

\section{Conclusion}

The feasibility of measuring the long-predicted phenomenon of real photon-photon scattering using the most intense lasers was investigated. Basing experimental parameters on the upcoming 10PW Vulcan upgrade, we found that splitting the beam into two counter-propagating pulses of differing intensity should be sufficient for measuring the elastic scattering process. Moreover, we found that the phenomenon was relatively robust, allowing for imperfect spatio-temporal alignment of the pulses. If the stronger of the two pulses can be compressed to sufficiently short durations, the vacuum four-wave mixing part of photon-photon scattering should in principle also be measurable. 


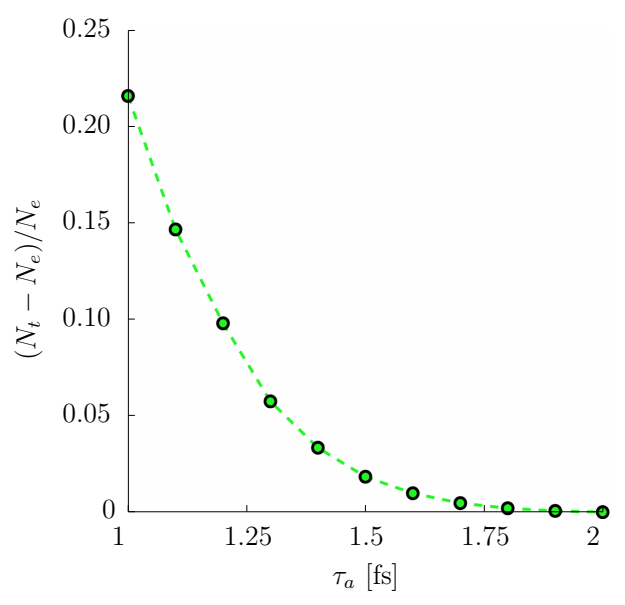

Figure 5: As the pulse duration $\tau_{a}$ of the strong pulse is reduced, the proportion of the total number of diffracted photons originating from vacuum four-wave mixing increases, plotted here for $P_{b}=10 / 3 \mathrm{PW}$, $P_{a}+P_{b}=10 \mathrm{PW}, \lambda_{a}=0.91 \mu \mathrm{m}, \lambda_{b}=0.2275 \mu \mathrm{m}, \tau_{b}=2 \mathrm{fs}, w_{a, 0}=0.91 \mu \mathrm{m}, w_{b, 0}=50 \mu \mathrm{m}, \boldsymbol{\varepsilon}_{a}=(1,0,0)$, $\boldsymbol{\varepsilon}_{b}=(0,0,1), \psi_{a}=\psi_{b}=0$ (adapted from [26]).

\section{References}

[1] W. Heisenberg and H. Euler. Photon acceleration in vacuum. Z. Phys., 98:714, 1936.

[2] V. Weisskopf. Über die Elektrodynamik des Vakuums auf Grund der Quantentheorie des Elektrons. Kgl. Danske Videnskab. Selskab, Mat.-fys. Medd., 14(6):6, 1936.

[3] F. Sauter. Z. Phys., 69:742, 1931.

[4] O. Halpern. Phys. Rep., 44:855, 1934.

[5] H. Euler and B. Kochel. Naturwissenschaften, 23:246, 1935.

[6] J. Schwinger. On gauge invariance and vacuum polarization. Phys. Rev., 82:664-679, 1951.

[7] V. Yanovsky et al. Ultra-high intensity- 300-TW laser at $0.1 \mathrm{~Hz}$ repetition rate. Opt. Express, 16:2109-2114, 2008.

[8] M. Marklund and P. K. Shukla. Nonlinear collective effects in photon-photon and photon-plasma interactions. Rev. Mod. Phys., 78(591):591, 2006.

[9] A. Di Piazza et al. Extremely high-intensity laser interactions with fundamental quantum systems. Rev. Mod. Phys., 84(1177):1177-1228, 2012.

[10] A. Ferrando et al. Nonlinear phase shift from photon-photon scattering in vacuum. Phys. Rev. Lett., 99(15):150404, Oct 2007.

[11] J. T. Mendonca et al. Photon acceleration in vacuum. Phys. Lett. A, 359:700-704, 2006.

[12] B. King, A. Di Piazza, and C. H. Keitel. Double-slit vacuum polarisation effects in ultra-intense laser fields. Phys. Rev. A, 82:032114, 2010.

[13] A. Di Piazza, K. Z. Hatsagortsyan, and C. H. Keitel. Light diffraction by a strong standing electromagnetic wave. Phys. Rev. Lett., 97(083603):083603, 2006.

[14] T. Heinzl et al. On the observation of vacuum birefringence. Opt. Commun., 267:318-321, 2006. 
[15] T. Heinzl and A. Ilderton. Exploring high-intensity QED at ELI. The European Physical Journal D, 55(2):359-364, 2009.

[16] B. King, A. Di Piazza, and C. H. Keitel. A matterless double-slit. Nature Photon., 4:92-94, 2010.

[17] G. Y. Kryuchkyan and K. Z. Hatsagortsyan. Bragg scattering of light in vacuum structured by strong periodic fields. Phys. Rev. Lett., 107:053604, Jul 2011.

[18] Y. Monden and R. Kodama. Enhancement of laser interaction with vacuum for a large angular aperture. Phys. Rev. Lett., 107:073602, Aug 2011.

[19] A. Di Piazza, K. Z. Hatsagortsyan, and C. H. Keitel. Harmonic generation from laser-driven vacuum. Phys. Rev. D, 72(085005), 2005.

[20] H. Ruhl and N. Elkina. Effects of quantum vacuum in ultra-intense laser fields and their simulation. Proc. SPIE, 8080:80801P-80801P-6, 2011.

[21] B. King, N. Elkina, and H. Ruhl. Photon polarisation in electron-seeded pair-creation cascades. Phys. Rev. A, 87(042117):042117, 2013.

[22] D. Bernard et al. Search for stimulated photon-photon scattering in vacuum, 2000.

[23] R Battesti and C Rizzo. Magnetic and electric properties of a quantum vacuum. Reports on Progress in Physics, 76(1):016401, 2013.

[24] H. Gies. Strong laser fields as a probe for fundamental physics. Eur. Phys. J. D, 55:311-317, 2009.

[25] K. Barth et al. Cast constraints on the axion-electron coupling. Journal of Cosmology and Astroparticle Physics, 2013(05):010, 2013.

[26] B. King and C. H. Keitel. Photon-photon scattering in collisions of laser pulses. New. J. Phys., 14(103002), 2012.

[27] D. Tommasini and Humberto Michinel. Light by light diffraction in vacuum. Phys. Rev. A (R), 82(011803):011803, 2010.

[28] G. Brodin, M. Marklund, and L. Stenflo. Proposal for detection of QED vacuum nonlinearities in Maxwell's equations by the use of waveguides. Phys. Rev. Lett., 87(171801):171801, 2001.

[29] E. Lundström et al. Using high-power lasers for detection of elastic photon-photon scattering. Phys. Rev. Lett., 96:083602, 2006.

[30] CLF Facilities Vulcan. Vulcan Glass Laser. http://www.clf.rl.ac.uk/Facilities/Vulcan/12248.aspx, 2013.

[31] R. Karplus and M. Neuman. Non-linear interactions between electromagnetic fields. Phys. Rev., 80:380-385, 1950.

[32] A Di Piazza. On refractive processes in strong laser field quantum electrodynamics. Ann. Phys., 338:302, 2013.

[33] B. King. Vacuum Polarisation Effects in Intense Laser Fields. PhD thesis, University of Heidelberg, 2010.

[34] M. Yeung et al. Near-monochromatic high-harmonic radiation from relativistic laser-plasma interactions with blazed grating surfaces. New. J. Phys., 15(025042), 2013. 